\title{
COMUNICACIÓN Y ECONOMÍA SOLIDARIA: CAMINOS COMUNES PARA EL CAMBIO SOCIAL
}

\section{Communication and Solidarity Economy: Common Paths for Social Change}

\section{Comunicação e economia solidária: caminhos comuns para a mudança social}

\author{
Quijano, Melba. Corporación DeMente Comunicación y Cultura para el cambio social \\ (Colombia) \\ melbaqui@gmail.com
}

\section{Fecha de recibido: 31 de enero de 2016 \\ Fecha de aceptado: 20 de marzo de 2016}

\section{RESUMEN}

En Colombia la economía social reúne a diversas organizaciones con distintas apuestas políticas y económicas. Por la especificidad jurídica como son constituidas, estas organizaciones pueden ser reconocidas como no gubernamentales; por ello, a este sector de la economía pertenecen las fundaciones, las corporaciones, las asociaciones y las tradicionales cooperativas, entre otras. Ante esta diversidad de organizaciones, se sugiere como tema central de este artículo la pregunta por el rol que la comunicación desempeña en las organizaciones de economía social y solidaria. Las reflexiones aquí expuestas surgieron de los resultados de la caracterización a organizaciones no gubernamentales que promueven el desarrollo social en los municipios de Bucaramanga y Barrancabermeja, en el departamento de Santander, Colombia. Como resultado de esta caracterización se destaca el concepto y acción de la comunicación que tienen las organizaciones, al tiempo que se evidenciaron algunos retos de las organizaciones de acuerdo con tres escenarios posibles: los procesos mediáticos para fortalecer la esfera pública; la acción 


\title{
organizacional de la comunicación; y la vinculación de la comunicación para el cambio social como apoyo en los procesos de empoderamiento y participación comunitaria.
}

Palabras clave: economía social, comunicación, cambio social.

\begin{abstract}
In Colombia the social economy brings together various organizations with different political and economic stakes. For the legal specificity of how they are formed, these organizations can be also recognized as nongovernmental, for this reason here can be foundations, corporations, associations and traditional cooperatives, among others.

Given this diversity of organizations, the question of the role communication can play in organizations of social economy suggested the central theme of this article. The reflections presented here emerged from the results of a characterization of non-governmental organizations promoting social development in the municipalities of Bucaramanga and Barrancabermeja, Santander State, Colombia.

As a result of this characterization, the concept of communication and action that organizations have highlighted. At the same time, some challenges were suggested by organizations according to three possible scenarios: media processes to strengthen the area public, organizational communication and action linking the field of communication for social change and supporting processes of empowerment and community participation.
\end{abstract}

Keywords: social economics, communication, social change.

\section{RESUMO}

$\mathrm{Na}$ Colômbia a economia social reúne diversas organizações com apostas políticas e económicas distintas. Pela especificidade jurídica de como se constituem, estas organizações podem ser reconhecidas igualmente como não governamentais, por isso a este setor da economia fazem parte das fundações, as corporações, as associações e as tradicionais cooperativas, entre outros.

Ante esta diversidade de organizações, a pergunta pelo papel que a comunicação pode desempenhar nas organizações de economia social e solidária se sugere como tema central deste artigo. As reflexões aqui expostas surgiram dos resultados de uma caracterização a organizações não governamentais que promovem o desenvolvimento social nos municípios de Bucaramanga e Barrancabermeja, no departamento de Santander, Colômbia.

Como resultado desta caracterização se destaca o conceito e ação da comunicação que têm as organizações, ao tempo que se evidenciaram alguns desafias sugeridos às organizações de acordo a três cenários possíveis: processos mediáticos para fortalecer a esfera pública, a ação organizacional da comunicação e a vinculação do campo da comunicação para mudança social como apoio aos processos de empoderamento e participação comunitária.

Palavras-chave: Economia social, comunicação, mudança social. 


\section{Introducción}

La comunicación para el cambio social se ha convertido en los últimos años en un campo de reflexión, desde el cual, la academia busca revisar los referentes teóricos presentes en ella. Conforme a esto, la investigación en este campo ha ganado proyección en el ejercicio de la academía, planteandose así dos escenarios posibles. El primero, definido desde las estructuras político-administrativas para la formulación del currículo de formación. El segundo escenario establece el reconocimiento de este campo para el análisis, la reflexión y la investigación-acción acerca de la comunicación relacionada con el desarrollo y el cambio social.

Este artículo tiene que ver con ese nuevo escenario investigativo en el cual el campo de la comunicación para el cambio social cobija el análisis de la comunicación en las organizaciones no gubernamentales que promueven el desarrollo social. Específicamente, el artículo analiza los resultados encontrados en la investigación denominada Caracterización del campo de la comunicación para el cambio social desde las organizaciones no gubernamentales que promueven el desarrollo social en Bucaramanga, el área metropolitana y Barrancabermeja, como municipio representativo de la región del Magdalena Medio ${ }^{1}$, llevada a cabo entre los años de 2012 y 2014.

Sobre el contenido del artículo, en primer lugar se exponen los conceptos asociados a la comunicación para el cambio social y la economía solidaria. Luego se contextualiza el proceso investigativo de la caracterización, sus resultados y la reflexión que en la comunicación estos generan. Se concluye con la exposición de los retos sugeridos para la comunicación en las organizaciones caracterizadas.

Para una mejor lectura del artículo, las organizaciones caracterizadas han sido relacionadas y descritas de manera general en un anexo. No obstante, vale la pena aclarar que, en general, las organizaciones caracterizadas promueven acciones de acompañamiento, formación y asesoría a procesos comunitarios encaminados a la promoción y la exigibilidad de los derechos humanos, económicos, sociales, culturales, sexuales y reproductivos.

Las prácticas sociales que promueven las organizaciones caracterizadas no son circunstanciales; por el contrario, han establecido una trayectoria de reflexión y acción en temas que bien podrían estar en el orden de comunicación y cultura (género, identidad, representación, memoria y patrimonio, etc.); comunicación y territorio (soberanía alimentaria, reforma agraria, producción agrícola, asentamientos urbanos, etc.) o, comunicación y cambio social (empoderamiento, tejido social, trabajo en red, incidencia política, etc.).

1 Bucaramanga es la ciudad capital del departamento de Santander en el oriente colombiano. "Lo definido como área metropolitana está conformada por los municipios de Bucaramanga, Floridablanca, Girón y Piedecuesta, pertenecientes a la provincia de Soto en el Departamento de Santander" (Área Metropolitana de Bucaramanga, s.f.). De otro lado, sobre el municipio de Barrancabermeja, este se encuentra "ubicado a $120 \mathrm{~km}$ al occidente de Bucaramanga, a orillas del Río Magdalena, en la región del Magdalena Medio, siendo el municipio más importante de ésta y segundo en todo el departamento" (Alcaldía de Barrancabermeja, 2013). 


\section{DISERTACIONES}

ESTUDIOS

Anuario electrónico de estudios en Comunicación Social

ISSN: 1856-9536

Doi: dx.doi.org/10.12804/revistas.urosario.edu.co/disertaciones/a.4529

Volumen 10, Número 1 / Enero-junio 2017

Versión PDF para imprimir desde

http://revistas.urosario.edu.co/index.php/disertaciones

\section{Punto de encuentro}

Cuando se piensa en economía social y solidaria, normalmente se hace la relación con las ciencias económicas y, en especial, con la experiencia de las cooperativas. Si bien esta relación es válida, en este artículo proponemos revisar otro punto de vista en el que se relacione la comunicación para el cambio social con el campo de acción de la economía social y solidaria, pensando a partir de las experiencias de organizaciones ubicadas en el oriente colombiano.

Tomamos como punto de partida lo estipulado en la Ley 454 de 1998, por medio de la cual es regulado el marco conceptual de la economía solidaria, donde, en el artículo 2 de esta ley, la economía solidaria quedó definida como "sistema socioeconómico, cultural y ambiental conformado por el conjunto de fuerzas sociales organizadas en formas asociativas identificadas por prácticas autogestionarias solidarias, democráticas y humanistas, sin ánimo de lucro para el desarrollo integral del ser humano como sujeto, actor y fin de la economía".

Bajo lo estipulado en la legislación colombiana, en el sector de la economía social y solidaria es posible encontrar diversos tipos de organizaciones entre cooperativas, fundaciones, corporaciones o asociaciones. Sin desconocer que existe una relación histórica de este concepto con la experiencia del sector cooperativo, en el contexto colombiano la economía social y solidaria se refiere a:

Todas aquellas formas asociativas solidarias y de ayuda mutua que se conocen como de economía colec-

tiva o de interés general. Concepción según la cual la economía social comprende la entidades de naturaleza cooperativa y empresas auxiliares, las organizaciones del movimiento mutualista, otras formas asociativas solidarias y de auto ayuda con fines económicos (Quijano y Reyes, 2004, p. 14).

De acuerdo con Alfonso Gumucio (2011), experto en el tema, este campo tiene que ver con las premisas y condiciones en las que se desarrolla. En cuanto a las premisas, siete en total, estas se asumen como presupuestos a tener en cuenta en la práctica de la comunicación para el cambio social. En relación con algunas de estas premisas, se destaca la apropiación del proceso por parte de los individuos o comunidades involucradas, y se resalta la intención de que las comunidades sean agentes de su propio cambio. En general, las premisas exponen el rechazo al modelo lineal de la comunicación como principio, al tiempo que valoran al proceso comunicativo, más que al producto.

Por otro lado, en lo concerniente a la Comunicación para el Cambio Social, esta se refiere a un campo de acción que une los aprendizajes obtenidos entre la comunicación para el desarrollo y la comunicación alternativa, la cual es reconocida como un proceso de diálogo y debate, basado en la tolerancia, el respeto, la equidad, la justicia social y la participación activa de todos (Gumucio, 2011, p. 32).

Como apoyo a la conceptualización de la comunicación para el cambio social, Gumucio plantea igualmente unas condiciones que, a su criterio, deben asumirse como indispensables para la comprensión de este campo. Estas condiciones son: participación comunitaria y apropiación; lengua y pertinencia cultural; generación de contenidos locales; uso de tecnología apropiada; y convergencia y redes. Es por esto que, entre los campos de acción de la economía social y solidaria y la práctica de la comunicación para el cambio social hay muchos puntos de encuentro, pues en la mayoría de los casos convergen experiencias colectivas basadas en la participación comunitaria como acción voluntaria y libre de quienes están involucrados. 


\section{DISERTACIONES}

ESTUDIOS

Anuario electrónico de estudios en Comunicación Social

ISSN: $1856-9536$

Doi: dx.doi.org/10.12804/revistas.urosario.edu.co/disertaciones/a.4529

Volumen 10, Número 1 / Enero-junio 2017

Versión PDF para imprimir desde

http://revistas.urosario.edu.co/index.php/disertaciones

Establecida la relación, vale la pena destacar tres elementos aclaratorios del desarrollo de la caracterización. El primero está en función del tiempo. Lo comprendido como diseño y trabajo de campo se realizó durante el segundo semestre de 2012 y el primer semestre de 2013. El análisis de la información se desarrolló durante todo el año 2013 para concluir el proceso investigativo y de entrega de resultados el primer semestre de 2014.

El segundo elemento aclaratorio tiene que ver con el por qué de una caracterzación y no de otro proceso investigativo. En general, una caracterización a diferencia de la sistematización, de la evaluación o incluso de los diagnósticos, no posee amplios referentes teóricos. El propósito y la metodología de una caracterización queda supeditada a las condiciones particulares de quien investiga o del objeto de la investigación. Una caracterización busca exponer las distintas características o condiciones en las que el objeto de la investigación se encuentra, en un momento determinado. Es como 'tomar una fotografía' a ese objeto social y evidenciar de esta las distintas lecturas que puedan ser posibles.

En este sentido, durante el desarrollo de esta caracterización, se propuso como reto la formulación de un marco teórico que guiara los objetivos y la metodología del proceso investigativo en relación con el campo de la comunicación para el cambio social. Así las cosas, entendiendo que este campo es relativamente nuevo, era probable no encontrar referentes en cuanto a otros procesos de caracterización similares. Y así sucedió, pues buena parte de las investigaciones halladas y consultadas se concentraban en evidenciar las condiciones laborales y las competencias académicas en el ejercicio del periodísmo. Paralelo al desempeño periodístico, otras investigaciones consultadas asumían como centro de análisis el desempeño del comunicador organizacional y, eventualmente, en algunas se planteaban análisis prospectivos del rol del comunicador y sus retos en la formación.

En general, el vacío de investigaciones que aborden la comunicación para el cambio social como campo de referencia del rol que tiene la comunicación en las organizaciones, fue el punto de partida ideal para justificar la realización de esta caracterización, pues con ella se establecería la evidencia de cómo este campo de acción es relevante para el desarrollo de las organizaciones no gubernamentales.

Finalmente, el tercer elemento aclaratorio sobre la caracterización tiene que ver con su realización, la cual llevó a gestionar y consolidar una base de datos de las organizaciones no gubernamentales presentes en el área de estudio. Como la lista superaba más de 300 organizaciones, fue necesario crear un filtro con el objeto social de cada organización y la vigencia de sus procesos. Bajo el rigor de este filtro se estableció una base de datos actualizada y completa de 81 organizaciones, de las que finalmente fueron seleccionadas 12 organizaciones. Las variables que definieron esta selección final fueron: el desarrollo de acciones de comunicación para el cumplimiento de su objeto social, y el consentimiento para ser objeto de esta caracterización.

A las organizaciones objeto de estudio se les aplicó un cuestionario de 24 preguntas que indagaba sobre 3 temas relevantes en la organización: 1) datos generales del entrevistado, 2) datos generales de la organización, y 3) datos de la comunicación en la organización. La aplicación del cuestionario requirió de un acercamiento preliminar a cada organización, y con ello socializar los propósitos de la caracterización.

Los resultados de la caracterización dan cuenta de reflexiones teórico-prácticas que permiten presentar las características generales de las organizaciones y sus formas particulares de definir, planear y ejecutar los procesos de comunicación. Indagar en las acciones desarrolladas en comunicación por parte de las organizaciones, requería de un enfoque cualitativo y descriptivo. Era necesario priorizar la información particular a cada organización y no la estandarización de sus procesos. Sobre este particular, los autores Palazzolo y Vidarte (2012) nos aclaran lo siguiente:

\section{3}




\section{DISERTACIONES}

ESTUDIOS

Anuario electrónico de estudios en Comunicación Social

ISSN: 1856-9536

Doi: dx.doi.org/10.12804/revistas.urosario.edu.co/disertaciones/a.4529

Volumen 10, Número 1/ Enero-junio 2017

Versión PDF para imprimir desde

http://revistas.urosario.edu.co/index.php/disertaciones

La discusión frecuente en el campo de las ciencias sociales se da en relación con los enfoques cualitativo y cuantitativo. La diferencia fundamental entre ambos es que el cuantitativo estudia la relación o asociación entre variables cuantificadas, y el cualitativo lo hace en contextos estructurales y situacionales. La investigación cualitativa trata de identificar la naturaleza profunda de las realidades, su sistema de relaciones, su estructura dinámica, y produce datos que comúnmente son considerados más "ricos y profundos”, no generalizables, en tanto están en relación con cada sujeto, grupo y contexto, con una búsqueda orientada al proceso (p. 88).

\section{Comunicación en la organización: resultados encontrados}

Desde el escenario de trabajo particular de cada organización, el tema de la comunicación fue abordado en relación con el concepto, la planeación, las acciones y la valoración sobre los procesos de documentación, memoria y sistematización. Con base en ello, y de manera general, se establecieron los siguientes resultados.

\section{¿Qué entienden las organizaciones por comunicación?}

El concepto de comunicación está mediado por la mirada funcionalista clásica, que lo determina a partir de los efectos producidos en el uso de un medio de comunicación. Es decir, a la comunicación se le da valoración operativa en cuanto a sus acciones mediáticas, especialmente impresas; como por ejemplo las acciones para la visibilidad mediática. No obstante, pese al paradigma mediático, algunas organizaciones encuentran la relación de su concepto con las acciones de participación, diálogo y acción en virtud de los procesos comunitarios. En este sentido, cabe destacar las definiciones dadas por el Comité Cultural de Zapamanga (Cocuza), el cual considera la comunicación como fundamental y transversal en la conformación de la organización:

- La comunicación es una dimensión humana, por lo cual es necesaria y vital para la existencia.

- La acción transversal referida a los proyectos que realiza la organización en acciones de educación e información.

- La acción de diálogo y encuentro entre diversos actores vinculados a un proyecto, en especial institucionales y comunitarios.

- Los procesos de creación y producción inmersos en el arte, los cuales son determinados por el encuentro, el diálogo y la pertinencia de sus acciones, de acuerdo con un contexto determinado.

- La base del ejercicio político fundamentado en la educación popular, de ahí que su concepto se relaciona con el de estrategia y capacidad para la incidencia.

- La comunicación como herramienta necesaria para la recuperación de la memoria y defensa del patrimonio.

- La noción política de la comunicación como un ejercicio mediático amparado en el derecho de la información y la democratización de la palabra. 


\section{¿Cómo desarrollan la planeación en comunicación?}

La mayoría de las organizaciones carecen de un proceso riguroso, decantado y ordenado de planeación en comunicación. Como resultado, las organizaciones asumen las acciones de comunicación de acuerdo con las circunstancias o coyunturas de los proyectos que están desarrollando.

Solo en la Corporación para la Construcción Participativa de la Salud Pública (Obusinga), se han dado experiencias de proyectos en las que el componente de comunicación fue analizado previamente con anterioridad a la formulación y ejecución del proyecto. Sumado a esta situación, las organizaciones carecen del seguimiento y evaluación necesarios para cualquier proceso de planeación en comunicación, por ello no cuentan con metodologías e instrumentos de evaluación que les permitan evidenciar los impactos o resultados obtenidos durante la ejecución de las acciones de comunicación.

A la pregunta realizada sobre el tema de planeación en comunicación, estos fueron los análisis realizados:

- Al no existir en los proyectos espacios de planeación para el componente de comunicación, normalmente quienes deciden este tipo de acciones priorizan los productos impresos y audiovisuales.

- En algunas organizaciones la comunicación asume un rol instrumental, por ello más que en el proceso, se piensa en acciones o productos, los cuales son definidos durante la ejecución de los proyectos de acuerdo con fechas particulares según los requerimientos que se van presentando relacionados con el objeto social, o el público objetivo de la organización.

- En el caso de la Agencia Prensa Rural, las acciones informativas para la producción mediática son definidas colectivamente en el espacio nacional de coordinación, conformado por los delegados de las organizaciones participantes.

- Generalmente, cuando las organizaciones han tenido la oportunidad de vincular a un profesional para desarrollar el tema de comunicación, prevalece el perfil de periodista más que un perfil integral de comunicación.

\section{¿Cuáles son las acciones que han desarrollado en comunicación?}

Consecuente con lo analizado en el tema de planeación, a la pregunta sobre las acciones desarrolladas en comunicación, las respuestas más recurrentes de las organizaciones fueron acciones mediáticas. Así, por ejemplo, para el caso de Prensa Rural, el tema de las acciones tiene que ver con la producción mediática, pues es a este propósito que debe su conformación. De otro lado, en algunas organizaciones como Fundación Mujer y Futuro, Corambiente y la Fundación CITU Experiencia Local, destaca la comunicación interpersonal como una acción clave y necesaria en sus procesos de formación, de acompañamiento y de fortalecimiento de la organización comunitaria.

En cuanto a la producción de medios (especialmente radio e impresos), estos tienen una fuerte relación con los procesos de educación que desarrolla cada organización. En este sentido, de las organizaciones que cuentan con recursos web 2.0, se destaca el uso de estas herramientas como apoyo a los procesos de registro y divulgación de los proyectos realizados y de sus producciones mediáticas. Así por ejemplo, el caso de Obusinga sobresale entre las demás organizaciones, en la medida que hace un uso creativo de su página web, desde la cual publica algunas de sus producciones como la serie radial Comer para vivir y el multimedia El Cesar se alimenta. También enlaza otros recursos desde la página web como el blog Diálogo alimentario-el Magdalena Medio visto desde la perspectiva alimentaria, así como el enlace con el blog de periodismo alimentario.

\section{5}




\section{DISERTACIONES}

ESTUDIOS

Anuario electrónico de estudios en Comunicación Social

ISSN: 1856-9536

Doi: dx.doi.org/10.12804/revistas.urosario.edu.co/disertaciones/a.4529

Volumen 10, Número 1 / Enero-junio 2017

Versión PDF para imprimir desde

http://revistas.urosario.edu.co/index.php/disertaciones

A manera de síntesis, a continuación algunas de las respuestas dadas sobre el tema de acciones en comunicación:

- Gestión y abogacía (advocacy) en medios masivos para la producción radial, televisiva e impresa de contenidos informativos relacionados con el objeto social o el público objeto de la organización.

- Apropiación de TIC y herramientas web (especialmente website, blog y Facebook) para los procesos de gestión y difusión de información, así como la visibilización (no publicitaria) de la organización.

- Comunicación interpersonal en procesos de capacitación y formación en temáticas afines al objeto social o al público objeto de la organización.

- Creación de estrategias lúdicas, didácticas y artísticas en procesos de educación y socialización en temáticas afines al objeto social o al público objeto de la organización.

- Aprovechamiento de recursos o medios alternativos como los parlantes comunitarios (chorote ${ }^{2}$ y y el voz a voz (radio bemba ${ }^{3}$ ), para la difusión de información y procesos de convocatoria.

- Vinculación y participación en procesos organizativos del tipo de redes para el fortalecimiento, y ampliación de las acciones de gestión e información.

- Procesos editoriales impresos para la divulgación de investigaciones.

- Convergencia informativa interinstitucional mediante la creación de una página web, que a manera de agencia de noticias, desarrolla información y relaciona los productos informativos en radio, prensa y tv, de acuerdo con el objeto social o con el público objeto de la organización.

- Creación artística (artes escénicas) y puesta en escena, basadas en el diálogo contextual entre la realidad y los sujetos participantes.

\section{¿Cuál es la valoración dada a las acciones de memoria, documentación y sistematización de sus procesos?}

El manejo de la información, para efecto de consolidar la memoria institucional de las organizaciones, representa un vacío común en la mayoría de ellas. Los registros de información son ocasionales y, en el mejor de los casos, la documentación solo se registra y organiza a manera de soportes para los informes de ejecución de los proyectos. La memoria institucional no es asumida como una prioridad en el registro y documentación de la historia de la organización. Hay organizaciones que cuentan con más de veinte años de acción, pero lamentablemente no existe un registro sobre las actividades iniciales, perdiéndose así la trayectoria del proceso realizado, sus metodologías particulares e incluso, los datos de quienes han participado o han sido aliados en sus procesos.

2 El chorote es una expresión coloquial que se usa en Colombia para referirse al altoparlante o las cornetas como recurso para amplificar sonido. Esta expresión es más usada en la zona andina colombiana, en donde es común ver en las torres de las iglesias cornetas que son utilizadas para emitir mensajes a la gente. "Los campanarios de los pueblos, las sedes de las juntas de acción comunal y los salones comunitarios están casi siempre coronados por una corneta. Su presencia puede pasar inadvertida hasta que se enciende y entonces toma posesión del entorno. Se impone" (Beltrán, 1996).

3 El voz a voz, como forma cotidiana de multiplicar la información con la palabra oral, se reconoce en el Caribe colombiano como radio bemba, expresión sugerida por Héctor Lavoe en su canción "Periódico de ayer". 


\section{DISERTACIONES}

ESTUDIOS

Anuario electrónico de estudios en Comunicación Social

ISSN: 1856-9536

Doi: dx.doi.org/10.12804/revistas.urosario.edu.co/disertaciones/a.4529

Volumen 10, Número 1 / Enero-junio 2017

Versión PDF para imprimir desde

http://revistas.urosario.edu.co/index.php/disertaciones

Sin duda, la trayectoria que tienen muchas de estas organizaciones y sus apuestas particulares en relación con su objeto social, dan cuenta de un número indeterminado de acciones. No obstante, muchas de estas acciones se han quedado en la memoria de quienes, circunstancialmente, han sido sus responsables. Las organizaciones reconocen una diferencia entre los procesos de memoria institucional y los procesos de sistematización. En este sentido, casi la mitad de las organizaciones caracterizadas valoran los procesos de sistematización, como un escenario útil de investigación social y además necesario para la evidencia, análisis y socialización de los aprendizajes de su práctica social.

En algunas organizaciones como la Fundación CITU Experiencia Local, Compromiso y Cocuza, reconocen las dimensiones histórica, epistemológica y de empoderamiento (Ramírez, 2008) que ofrece cualquier proceso de sistematización. No obstante, son conscientes de que estos procesos requieren de tiempo, recursos y decisión institucional para su desarrollo. Solo la Corporación Obusinga y la Fundación de la Mujer, han desarrollado acciones en esta índole al margen de la ejecución de algunos proyectos.

En general, las respuestas ofrecidas al tema de documentación, memoria y sistematización, están representadas en los siguientes análisis:

- Débiles procesos de registro de la memoria institucional de los proyectos realizados y de las acciones relevantes en la organización; no obstante, algunas organizaciones documentan rigurosamente la información relacionada con los proyectos financiados.

- Algunas organizaciones presentan confusión entre los procesos de sistematización y de documentación relacionados con las acciones de rendición de cuentas e informes de gestión de proyectos.

- Utilización del recurso web 2.0 (website) para la publicación de productos mediáticos (videos, series radiales y periódicos) como recurso de registro y visibilización de la memoria institucional en proyectos de animación sociocultural.

- Producción de material educativo y documental con un doble propósito: la formación y la documentación de las acciones relevantes al proyecto.

- Reconocimiento de metodologías y aprendizajes obtenidos durante la práctica social aunque no estén documentados.

- Valoración y distinción del proceso de creación y de sistematización como dos momentos relevantes en la organización, pero esto solo se tiene en cuenta al momento de creación donde se le reconoce mayor concentración y dedicación.

- Reconocimiento del relevo generacional y de la rotación de personal, como factores decisivos en la pérdida de información y en la carencia de documentación sobre las experiencias.

\section{Concepto y acción de la comunicación: reflexiones sugeridas}

La práctica comunicativa que las organizaciones caracterizadas desarrollan puede tomarse como referente para un ejercicio analítico de los diferentes marcos teóricos que la comunicación para el cambio social asume. En este sentido, algunos de estos elementos teóricos están relacionados con temas como: la Investigación-Acción Participativa (IAP), los medios radicales, la pedagogía de la comunicación y los medios ciudadanos.

A continuación se plantean algunas reflexiones teóricas relacionadas con estos temas y con lo encontrado durante la caracterización. 


\section{DISERTACIONES}

ESTUDIOS

Anuario electrónico de estudios en Comunicación Social

ISSN: $1856-9536$

Doi: dx.doi.org/10.12804/revistas.urosario.edu.co/disertaciones/a.4529

Volumen 10, Número 1 / Enero-junio 2017

Versión PDF para imprimir desde

http://revistas.urosario.edu.co/index.php/disertaciones

\section{Investigación-Acción Participativa (IAP) para la comunicación}

Obusinga, e incluso Cocuza, han desarrollado como metodología particular de sus procesos la Investigación-Acción Participativa (IAP) procurando que en sus investigaciones, la participación comunitaria sea voluntaria y que esta sirva como fuente de información para reconocer la dimensión de sus problemas sociales. Por ejemplo, en el desarrollo de las investigaciones sobre recuperación de la memoria alimentaria y las canastas alimentarias básicas $(\mathrm{CAB})^{4}$, Obusinga primero establece un acercamiento comunitario, para que a partir de este, se defina el grupo que desarrollará la investigación y del cual, posteriormente, se establecerán las acciones para la socialización de los resultados, incluyendo, acciones de producción mediática y de interlocución con las instituciones del Estado. Obusinga establece para cada proceso investigativo la creación de instructivos metodológicos que son dispuestos de manera general a todas las personas participantes, e incluso, para los visitantes de su página web es fácil encontrar estos recursos. En su página web, también se encuentran publicados los resultados de algunas de estas investigaciones. En general, la apropiación que hace Obusinga sobre el tema de la IAP es transversal a todo su proceso de acompañamiento comunitario. Desarrollo de cartografía alimentaria, recuperación de memoria alimentaria, inventario de acciones de autonomía alimentaria y sondeos de mercado, constituyen la oferta de investigaciones que dinamiza Obusinga a partir de la IAP.

\section{Medios radicales}

Es una categoría de acción reconocida por John Downing en aquellos medios que usualmente expresan visiones alternativas frente a los proyectos hegemónicos establecidos en las sociedades. Para Downing (2011), estos medios sirven a dos propósitos fundamentales, puesto que, por una parte "expresan opinión vertical desde los espacios subordinados directamente contra las estructuras de poder y sus formas de comportamiento. [Y por otra parte,] construyen de manera horizontal apoyo, solidaridad y trabajo en red contra las políticas o aun contra la supervivencia de la estructura de poder imperante" (p. 406).

Los procesos mediáticos y las acciones de comunicación que desarrollan Prensa Rural y la Corporación de Objetores de Conciencia-Quinto Mandamiento encuentran su representación en la denominación y características que Downing otorga al ejercicio de los medios radicales. Prensa Rural, por su parte, hace uso de su plataforma mediática para divulgar las denuncias contra la violación de derechos humanos de las organizaciones agrarias y campesinas; igualmente, utiliza sus medios para defender los procesos comunitarios desarrollados en las zonas

4 Sobre el proyecto de recuperación de la memoria alimentaria Obusinga afirma que "está basada en metodologías provenientes de la historia oral, la recuperación de la memoria alimentaria es una metodología fundamentada en la activación de los recuerdos de las distintas generaciones y el levantamiento de testimonios sobre la evolución de la alimentación de los pobladores del municipio a lo largo del tiempo. A partir de allí, las comunidades mejoran su capacidad de construir visiones de futuro alimentario y crear proyectos de vida colectivos". Así mismo, sobre la canasta alimentaria básica, aclara que "es el estudio más completo de Seguridad Alimentaria y Nutricional que se realiza a escala municipal. Consiste en una encuesta de hogares que indaga el acceso y consumo de alimentos por parte de las familias. La CAB toma ente 4 y 6 meses para su realización y permite conocer los niveles de hambre crónica y hambre oculta que hay en el municipio, así como la dinámica del mercado local de alimentos". 


\section{DISERTACIONES}

ESTUDIOS

Anuario electrónico de estudios en Comunicación Social

ISSN: 1856-9536

Doi: dx.doi.org/10.12804/revistas.urosario.edu.co/disertaciones/a.4529

Volumen 10, Número 1 / Enero-junio 2017

Versión PDF para imprimir desde

http://revistas.urosario.edu.co/index.php/disertaciones

de reservas campesinas y, en general, le siguen 'el pulso' a las protestas de los diversos movimientos sociales contestatarios y de oposición presentes actualmente en el país y en América Latina.

A su vez, en Quinto Mandamiento, las prácticas de comunicación se relacionan con los medios radicales en sus formas particulares de expresión, basándose en la música popular, el arte callejero y las protestas sociales artísticas, como las desarrolladas con el nombre de 'contra marcha' durante los desfiles militares del 20 de julio.

[...] durante el desfile entregamos los globos a la gente. Hay un caso curioso, un policía que pide el globo se la da a su niño y cuando ve que tenía un mensaje lo lee y se lo revienta, los mensajes eran como "ningún ejército defiende la paz", "deja las armas ven a jugar", "con lo que cuesta un tanque podemos alimentar un barrio", "no más inversión para la guerra más inversión para la vida", "más educación, salud, menos guerra". Más o menos unos 10 a 12 mensajes, más de 100 globos (Arias, 2013).

Downing señala que los medios radicales se refieren igualmente a una serie de expresiones no tecnológicas, enmarcadas dentro de diversas manifestaciones artísticas. Al respecto el autor afirma:

La danza, el teatro de la calle, las historietas ilustradas, los afiches, la parodia, la sátira, el performance, el graffiti, los murales, las canciones populares y la música instrumental son [...] solo algunas de las formas más obvias de los medios radicales cuyo empuje comunicativo no depende de una lógica argumentada rigurosamente, sino de su fuerza estéticamente concebida y concentrada (Downing, 2008, p. 1112).

\section{Pedagogía de la comunicación o el enfoque freiriano}

La comunicación, comprendida como acción de diálogo, de voluntad, de participación, de conocimiento conjunto, ha sido fortalecida en estos conceptos a partir de los diversos aportes que América Latina ha ofrecido en su vasta experiencia de las prácticas comunitarias de comunicación. Por esta razón, cobra relevancia la relación directa que ofrece la experiencia del Centro Cultural Horizonte Ciudadela Educativa, al considerar su enfoque de trabajo desde el diálogo contextual con la gente, "con este interrogante grande en donde el grupo de trabajo es el que más demanda, el que dice ¿quién soy?" (Ripamonti, 2013).

En las acciones que desarrolla el Centro Cultural, ese diálogo contextual es transversal a la creación artística, en la medida que, como proceso pedagógico, se indaga sobre el rol, imaginarios y confrontaciones que representan la vivencia de los jóvenes de la comuna 7 de Barrancabermeja, territorio en donde se concentra el Centro Cultural. Sobre este particular su director afirma:

El arte tiene que tener ese lugar, el lugar de encuentro, que la comunidad se encuentre, que los jóvenes se encuentren, se descubran encontrándose entre sí y con la comunidad. Si los jóvenes logran entrar en diálogo con la comunidad logran su pertinencia, en esto del diálogo con la comunidad, entonces la provocación juvenil es también sana. Si se logra que sea en diálogo con la comunidad, entonces ese caos que en el arte tiene un lugar estupendo de diálogo, es parte fundamental de lo social, porque llena de imágenes y de provocaciones a una sociedad que necesita esas provocaciones, porque si no sigue repitiéndose así mismo (Ripamonti, 2013).

De igual manera, la consideración por la educación popular y los procesos territoriales que demandan una pedagogía de la comunicación basada en el diálogo, representa el trabajo de Cocuza. Esta organización, establecida en el barrio Zapamanga III etapa en Floridablanca, valora la educación popular como clave de su trabajo 


\section{DISERTACIONES}

ESTUDIOS

Anuario electrónico de estudios en Comunicación Social

ISSN: 1856-9536

Doi: dx.doi.org/10.12804/revistas.urosario.edu.co/disertaciones/a.4529

Volumen 10, Número 1 / Enero-junio 2017

Versión PDF para imprimir desde

http://revistas.urosario.edu.co/index.php/disertaciones

comunitario, acciones igualmente reconocidas como herencia de la influencia que desarrollara en la organización la teología de la liberación.

En relación con esto, el coordinador del área cultural de Cocuza, afirma:

La metodología que utilizamos aquí es la educación popular, entonces partimos del saber de los otros. La forma de enseñar también parte de la cotidianidad, usamos las herramientas o las formas de vida que las personas tienen para, a partir de eso reflexionar y formarnos en ciertos temas. Entonces lo comunicativo, es que tratamos de reflejar la realidad lo más cercano que se pueda, para que se sientan identificados (Palacios, 2013).

Conforme a lo anterior, el reconocimiento del diálogo contextual, crítico y reflexivo como base de una comunicación que promueva el cambio social y el desarrollo está relacionado con los postulados de Freire, en la medida que se establece una pedagogía de la comunicación hacia una educación problematizadora.

Freire piensa en una pedagogía de la comunicación como algo que podría establecer un diálogo y proporcionar un nuevo contenido programático a la educación. La clave de este sistema no estaría en la alfabetización en sí misma, sino en superar antes la interpretación mágica o ingenua del mundo, en desarrollar una comprensión crítica (Marqués de Melo, 2008, p. 279).

\section{Medios ciudadanos}

Desde las diversas concepciones existentes para analizar la producción y el propósito de los medios masivos de comunicación distintos a los comerciales, ha sido recurrente relacionar en un solo concepto la noción de medio alternativo, participativo o comunitario. Por mucho tiempo, los considerados 'otros medios' en América Latina fueron definiéndose y estandarizándose alrededor de una idea común de medios de oposición, contestatarios, contrahegemónicos e incluso clandestinos, con lo cual, el campo de acción de muchos de estos medios quedaba reducido a una concepción binaria de poder. En ese largo transitar de debates académicos alrededor de la praxis comunicativa latinoamericana, Clemencia Rodríguez hace una aportación fundamental, considerando el tránsito de la definición de medios alternativos a medios ciudadanos, por lo cual asume este último, desde las tesis de democracia radical propuesta por Chantal Mouffe y desde las nociones de comunicación y cultura asumidas por Martín-Barbero.

Por lo tanto, los "medios ciudadanos" son aquellos que facilitan la transformación de individuos y comunidades en aquello que Mouffe llama "ciudadanos" y que Martín Barbero define como subjetividades empoderadas, con voz propia. Los medios ciudadanos son emisoras, televisiones comunitarias, iniciativas de Internet, todos aquellos medios que abren espacios de comunicación donde hombres, mujeres, niñas y niños aprenden a manipular sus propios lenguajes, códigos, signos y símbolos, y adquieren poder para nombrar el mundo en sus propios términos. Los medios ciudadanos activan procesos a través de los cuales individuos y comunidades re-codifican tanto sus contextos como su propia identidad. En últimas, estos procesos mediáticos brindan a los ciudadanos la oportunidad de transformar sus identidades en subjetividades empoderadas, fuertemente ancladas en sus culturas locales e impulsadas por utopías bien definidas. Los medios ciudadanos son los medios que usan los ciudadanos para activar procesos de comunicación que contribuyen a moldear sus comunidades (Rodríguez, 2009, p. 19). 


\section{DISERTACIONES}

ESTUDIOS

Anuario electrónico de estudios en Comunicación Social

ISSN: $1856-9536$

Doi: dx.doi.org/10.12804/revistas.urosario.edu.co/disertaciones/a.4529

Volumen 10, Número 1 / Enero-junio 2017

Versión PDF para imprimir desde

http://revistas.urosario.edu.co/index.php/disertaciones

Esta definición de medios ciudadanos, como término y no como categoría, permitiría interpretar mejor los procesos que en comunicación ha desarrollado la Fundación CITU Experiencia Local y la Corporación Compromiso. Así, por ejemplo, la experiencia de Telegrama Comunitario desarrollado en asentamientos urbanos de los municipios de Girón y Piedecuesta durante el año 2012 por la Fundación CITU Experiencia Local, permitió que la comunidad participante, en uso de los recursos web 2.0, asumiera un rol activo de ciudadanía al permitirse analizar e intervenir en los procesos de planeación territorial de sus asentamientos. Desde el Telegrama Comunitario, los participantes de esta iniciativa, en su mayoría jóvenes, fueron protagonistas de decisiones y acciones de diseño urbanístico, por lo cual hacían uso de las redes sociales digitales para promover el reconocimiento de las condiciones territoriales de los asentamientos y socializar algunas condiciones de riesgo ante las autoridades competentes.

En lo que respecta a Compromiso, su relación como medio ciudadano se establece específicamente en la experiencia de la emisora comunitaria La Brújula, cuyo concesionario es precisamente la Corporación. Como medio, su parrilla de programación, y la colectividad que producen estos programas radiales, tiene relación con lo que Rodríguez (2008) establece como características de un medio ciudadano.

Hablar de "medios ciudadanos" implica: primero, que una colectividad asume el rol de su ciudadanía al intervenir y transformar activamente el panorama mediático ya establecido; segundo, que estos medios desafían los códigos sociales, las identidades legitimadas y las relaciones sociales institucionalizadas; y tercero, que estas prácticas comunicacionales les confieren poder a la comunidad involucrada, en la medida en que estas transformaciones y cambios son posibles (p. 1143).

\section{Retos de la comunicación en las organizaciones caracterizadas}

Cada organización caracterizada, por distinta que parezca su objeto social o su público, tiene en común la intención de fomentar la construcción de una ciudadanía activa, crítica y empoderada en sus derechos. Así por ejemplo, la objeción de conciencia al servicio militar, la exigibilidad de derechos de las organizaciones agrarias y campesinas, la ciudadanía e imaginarios juveniles, la memoria y representación, la defensa de los derechos humanos, la seguridad y la autonomía alimentaria, la participación ciudadana en la planeación territorial y los enfoques diferenciales de género, representan en conjunto algunos de los elementos que hoy día abogan por un proyecto ideal de sociedad.

Sin pretender establecer un 'deber ser' de los procesos de comunicación en la organización, en este apartado se sugieren algunas reflexiones y acciones de cómo podrían desarrollarse dichos procesos. No obstante, es importante destacar que cada organización requiere de una mirada puntal de sus procesos y acciones en comunicación, pues poseen objetos sociales distintos. Lo planteado como desafíos de la comunicación en la organización se presenta como una orientación para mediar saberes particulares entre la academia y las organizaciones sociales. La propuesta se basa en asumir la planeación participativa de los procesos de comunicación a partir de una reflexión rigurosa sobre el sentido de la comunicación en la organización.

Lo sugerido como planeación participativa en el campo de la comunicación no es nuevo. De hecho, es una propuesta de acción surgida a partir de la experiencia de las radios comunitarias en América Latina, las cuales han decantado este proceso participativo en la construcción de sus proyectos comunicativos, como documento estratégico que guía la acción política del medio. En este sentido, se sugiere aquí establecer la realización de un documento similar para cada organización. Un documento que le permita a cada organización pensar los procesos estratégicos

\section{1}




\section{DISERTACIONES}

ESTUDIOS

Anuario electrónico de estudios en Comunicación Social

ISSN: 1856-9536

Doi: dx.doi.org/10.12804/revistas.urosario.edu.co/disertaciones/a.4529

Volumen 10, Número 1 / Enero-junio 2017

Versión PDF para imprimir desde

http://revistas.urosario.edu.co/index.php/disertaciones

de comunicación. Un documento que proponemos llamar como 'de marco de acción' o 'de lineamientos estratégicos para la comunicación', el cual debe ser construido de manera particular para cada organización, como resultado de una reflexión conjunta de quienes participan de la organización.

Se trata entonces de un documento de construcción colectiva en el que sean analizados los diferentes campos de acción de la organización y su posible relación con la comunicación, por lo cual se sugieren tres áreas de acción que pueden resultar estratégicas para cada organización y que han sido propuestas a partir de los resultados de la caracterización. Estas son: 1) los procesos mediáticos para fortalecer la esfera pública; 2) la comunicación y su acción en la organización; 3) y la comunicación para el cambio social como apoyo a los procesos de empoderamiento y participación comunitaria.

\section{La conquista de la esfera pública}

Algunas organizaciones han realizado procesos para lograr su visibilidad en la agenda mediática local y regional, pero en algunos casos, estos procesos de visibilidad han demandado de una acción de lobby mediático, que resulta excesivamente costoso para las organizaciones. No obstante, la visibilidad mediatizada se considera necesaria para el reconocimiento de sus proyectos. Conquistar la esfera pública, requiere ante todo de un manejo estratégico y continuo de la agenda informativa que las organizaciones deben empezar a orientar. Toda organización social desarrolla acciones que merecen ser reconocidas y visibilizadas, por ello pueden hacer un agenciamiento informativo de estas ante los medios locales, o sus propios medios, en la medida que son acciones de interés público y general.

Para incidir en la agenda pública mediática, podrían considerarse estas acciones:

- Aprovechamiento de los distintos recursos web 2.0, en la medida que esta web ha sido considera la web para el desarrollo, pues a partir de ella, las organizaciones acceden a estos recursos de manera gratuita, generan contenidos compartidos y desarrollan plataformas técnicas para la visibilidad y el fortalecimiento de sus procesos en red (Ashley, Kenton y Milligan, 2009).

- Construcción de una base de datos actualizada y continua de medios y periodistas.

- Generación periódica de boletines de prensa para la divulgación y promoción de sus acciones.

- Fomentar el desarrollo de estrategias como el media advocacy (abogacía de medios) y de periodismo ciudadano para fortalecer procesos de debate y de reflexión que promuevan una agenda informativa local incluyente y pluralista. De manera especial, la estrategia de periodismo ciudadano sugeriría que dichas organizaciones establezcan alianzas con medios distintos a los convencionales, es decir, medios de interés público, comunitarios o incluso privados.

\section{La comunicación y su acción en la organización}

En las organizaciones caracterizadas hay una baja valoración de las acciones de comunicación organizacional, ya que son consideradas como propias de otras organizaciones de corte neoliberal o comercial. Sin duda, esta 


\section{DISERTACIONES}

ESTUDIOS

Anuario electrónico de estudios en Comunicación Social

ISSN: 1856-9536

Doi: dx.doi.org/10.12804/revistas.urosario.edu.co/disertaciones/a.4529

Volumen 10, Número 1 / Enero-junio 2017

Versión PDF para imprimir desde

http://revistas.urosario.edu.co/index.php/disertaciones

apreciación contradice las perspectivas actuales de la comunicación organizacional ${ }^{5}$, en la medida que toda organización, independiente de su objeto social o del número de participantes, requiere de la formalización de canales externos e internos de comunicación y de información, a partir de los cuales se fortalezca la cultura organizacional, las relaciones interinstitucionales y los procesos de posicionamiento de la marca. Si las organizaciones de la economía social y solidaria accedieran a analizar el manejo de sus procesos de información, con seguridad fortalecerían sus dinámicas internas e incluso, ayudarían a fomentar y fortalecer las relaciones públicas y las alianzas interinstitucionales.

A continuación, se sugieren algunas acciones de fácil aplicabilidad para los procesos de la comunicación organizacional:

- Realización de un diagnóstico sobre la existencia y condiciones de canales externos e internos de comunicación e información con los diversos públicos de la organización.

- De tener recursos web u otros medios, realizar procesos de caracterización de dichos medios con lo cual, no solo se deje evidencia de las condiciones de estos recursos, sino que también se propicie un análisis sobre su uso, apropiación y condiciones particulares.

- Aprovechamiento de los recursos web 2.0 como plataforma de visibilidad y registro de los procesos desarrollados por la organización.

- Construcción de la memoria institucional de la organización, en la cual se presente un registro ordenado de recursos audiovisuales y escritos sobre las distintas acciones desarrolladas por la organización.

- Fortalecimiento, en cuanto a periodicidad y contenido, de los productos informativos internos, como plegables, periódicos e incluso carteleras informativas, entre otros.

\section{La comunicación para el cambio social desde las organizaciones}

El campo de la comunicación para el cambio social se plantea como tema central para la formulación del documento 'de marco de acción' o ‘de lineamientos estratégicos para la comunicación' propuesto para cada organización. Por esta razón, desde esta línea, se propone pensar las acciones de comunicación y participación como un proceso que requiere la planeación y la acción de quienes se asumen como beneficiarios de los procesos; es decir, propiciar la participación, de manera consciente, crítica y voluntaria, como una condición sine qua non de las acciones sugeridas desde el campo de la comunicación para el cambio social. El diseño de una estrategia de 'eduentretenimiento', la producción de una serie radial, la sistematización de experiencias, la apropiación social de los medios, la puesta en escena de un proceso artístico, el desarrollo de piezas audiovisuales y hasta la creación de pedagogías para la comprensión -no persuasión - de cambios en actitudes, comportamientos y percepciones, serían entre otros, los posibles desempeños que se podrían sugerir a las organizaciones caracterizadas.

5 Según Mónica del Valle (2003), “la nueva visión de la comunicación organizacional debe partir del supuesto de que las empresas son realidades en construcción, que permiten visiones integrales y posibilidades de intervenciones deliberadas y sistemáticas para adecuarlas a lo que pretenden ser. Se debe pensar en la Comunicación Organizacional como una estrategia integral que posibilita proyectar identificadores para propiciar una imagen coherente de la organización, relacionar sus necesidades e intereses con los de su personal, con los consumidores, con el contexto en el que actúa y con las necesidades sociales" (p.1).

\section{3}


En la comunicación para el cambio social no existen fórmulas predeterminadas para establecer acciones, pues estas deben ser particulares a cada proceso. Lo que existe, son una serie de referentes de la práctica social, reflexiones teóricas y sobre todo, las llamadas premisas y condiciones sugeridas por Gumucio (2011). Conforme a lo anterior, de asumirse el campo de la comunicación para el cambio social como un escenario útil para el trabajo comunitario en las organizaciones, estas tendrían que 'desinstrumentalizar' la comunicación, para mirarla en función del proceso y no del producto. Así mismo, tendrían como reto 'desintrumentalizar' la participación en los procesos de comunicación, para no repetir los equívocos que la comunicación para el desarrollo -escuela funcionalista norteamericana- estableció para América Latina desde el modelo de la difusión de innovaciones.

\section{Conclusiones}

La caracterización permitió evidenciar una serie de aprendizajes que cada organización tiene acerca del quehacer de la comunicación en sus procesos, los cuales se constituyen en temas relevantes para futuras investigaciones. De otro lado, a partir de los resultados de la caracterización, fue claro determinar que los procesos de planeación y acción de comunicación pasan desapercibidos en la mayoría de las organizaciones. La valoración que la dan al campo de acción de la comunicación queda limitada a los productos mediáticos, que surgen casi siempre como acciones de visibilidad o como alternativas mediáticas para la educación.

Bajo este panorama, vale la pena destacar que este tipo de investigación tiene importancia para la academia, porque es imprescindible el reconocimiento de los elementos del contexto social, para que a partir de estos la oferta académica tenga sentido y coherencia con las demandas de dicho contexto.

Finalmente, para las organizaciones pertenecientes a la economía solidaría, las sugerencias hechas en la caracterización pueden ser fácilmente implementadas. Así mismo, basadas en las evidencias de estos procesos de caracterización, las organizaciones podrán analizar de manera colectiva conceptos y acciones sobre el sentido particular que debe tener la comunicación en sus procesos organizativos y comunitarios.

\section{Referencias}

1. Alcaldía de Barrancabermeja. (2013). Presentación. Barrancabermeja. Recuperado de https://www. barrancabermeja.gov.co/Institucional/Paginas/Institucional.aspx

2. Área Metropolitana de Bucaramanga. (s.f.). Plan de gestión integral de residuos sólidos del área metropolitana de bucaramanga. Recuperado de http://www.bucaramanga.gov.co/documents/dependencias/ DIAGNOSTICO\%20FINAL\%20COMPILADO.pdf

3. Arias, J. (2013). Presidente saliente Corporación de Objetores y Objetoras por conciencia Quinto Mandamiento. Entrevistador: Melba Quijano. Grabación digital. Barrancabermeja.

4. Ashley, H., Kenton, N. y Milligan, A. (2009). Change and Hand: Web 2.0 for Development. Participatory, Learning and Action. Recuperado de http://pubs.iied.org/pdfs/14563IIED.pdf

5. Beltrán, M. (1996). Los caminos de la radio comunitaria. Ministerio de Comunicaciones: Proyecto de comunicación para la infancia y la mujer - Proyecto Enlace. Editora Guadalupe Ltda.

\section{4}




\section{DISERTACIONES}

ESTUDIOS

Anuario electrónico de estudios en Comunicación Social ISSN: 1856-9536

Doi: dx.doi.org/10.12804/revistas.urosario.edu.co/disertaciones/a.4529

Volumen 10, Número 1 / Enero-junio 2017

Versión PDF para imprimir desde

http://revistas.urosario.edu.co/index.php/disertaciones

6. Del Valle, M. (abril-mayo, 2003). La comunicación organizacional de cara al siglo XXI. Revista Razón y Palabra, (32). Recuperado de http://www.razonypalabra.org.mx/anteriores/n32/mvalle.html

7. Downing, J. (2008). Comunidad, democracia, diálogo y medios radicales. En A. Gumucio. y T. Tufte. (comps.), Antología de la comunicación para el cambio social: lecturas históricas y contemporáneas. (pp. 1103-1114). La Paz: Consorcio de Comunicación para el cambio social/ Plural Editores.

8. Downing, J. (2011). Medios radicales. Comunicación rebelde y movimientos sociales. En J. Pereira. y A. Cadavid. (eds.), Comunicación, desarrollo y cambio social. Interrelaciones entre comunicación, movimientos ciudadanos y medios. (pp. 399-447). Bogotá: Pontificia Universidad Javeriana.

9. Gumucio, A. (2011). La comunicación para el cambio social: clave del desarrollo participativo. En J. Pereira y A. Cadavid. (eds.), Comunicación, desarrollo y cambio social. Interrelaciones entre comunicación, movimientos ciudadanos y medios. (pp. 19-35). Bogotá: Pontificia Universidad Javeriana.

10. Congreso de la República de Colombia. (1998). Ley 454 de 1998, "Por la cual se determina el marco conceptual que regula la economía solidaria, se transforma el Departamento Administrativo Nacional de Cooperativas en el Departamento Nacional de la Economía Solidaria, se crea la Superintendencia de la Economía Solidaria, se crea el Fondo de Garantías para las Cooperativas Financieras y de Ahorro y Crédito, se dictan normas sobre la actividad financiera de las entidades de naturaleza cooperativa y se expiden otras disposiciones". Bogotá: Diario oficial No. 43.357, agosto 4 de 1998.

11. Marqués de Melo, J. (2008). La comunicación en la pedagogía de Freire. En A. Gumucio y T. Tufte. (comps.), Antología de la comunicación para el cambio social: lecturas históricas y contemporáneas. (pp. 278-292). La Paz: Consorcio de Comunicación para el cambio social/ Plural Editores.

12. Palazzolo, F. y Vidarte, V. (2012). Claves para abordar el diseño metodológico. En M. Silvina et al. (eds.), Hacia la tesis. Itinerarios conceptuales y metodológicos para la investigación en comunicación. (pp. 83-92). La Plata: Universidad Nacional de La Plata.

13. Palacios, L. (2013) Coordinador área cultural Cocuza. Entrevistador: Lizeth León. Grabación digital. Floridablanca.

14. Quijano, J. y Reyes, M. (2004). Historia y doctrina de la cooperación. Universidad Cooperativa de Colombia. Bogotá: Universidad Cooperativa de Colombia.

15. Ramírez, J. (2008). La sistematización de experiencias de innovación educativa: una posibilidad de producción de conocimientos pedagógicos. (Tesis de pregrado inédita). Universidad Pedagógica Nacional, Bogotá.

16. Ripamonti, G. (2013). Director centro cultural horizonte ciudadela educativa. Entrevistador: Melba Quijano. Grabación digital. Barrancabemeja.

17. Rodríguez, C. (2008). De medios alternativos a medios ciudadanos. En A. Gumucio y T. Tufte. (comps.), Antología de la comunicación para el cambio social: lecturas históricas y contemporáneas. (pp. 1131-1148). La Paz: Consorcio de Comunicación para el cambio social/ Plural Editores.

18. Rodríguez, C. (diciembre, 2009). De medios alternativos a medios ciudadanos: trayectoria teórica de un término. Revista de la Facultad de Comunicaciones de la Universidad de Antioquia, (21), 13-25. 


\section{Anexo: Las organizaciones caracterizadas}

Cada organización seleccionada responde a un objeto social, metodologías y públicos diversos. En algunas, sus campos de acción se hacen comunes a otras; no obstante, para no errar en una clasificación estandarizada, a continuación se expone un cuadro de presentación con los datos característicos de cada organización, al tiempo que se abordan algunos elementos generales del proceso de comunicación que desarrollan.

\section{Organizaciones caracterizadas en el municipio de Barrancabermeja}

Centro Cultural Horizonte Ciudadela Educativa: es una corporación que enfoca su trabajo en procesos artísticos y pedagógicos basados en las artes escénicas. Apoyados por voluntarios, su apuesta metodológica se concentra en el diálogo contextual y asume los principios de la pedagogía para la liberación promovidos por Freire. La creación artística es el resultado de un ejercicio de concientización, comprensión y conocimiento que fortalece el proyecto de transformación social, haciendo uso de las artes escénicas. http://www.etpbarranca.org/comunicacion/index.html

Agencia Prensa Rural: es una plataforma mediática de recursos web que algunas organizaciones campesinas usan para su acción colectiva. Es un proceso organizativo tipo red, en el que sus acciones informativas son definidas colectivamente en el espacio nacional de coordinación, conformado por delegados de las diferentes organizaciones participantes. Su agenda temática se centra exclusivamente en difundir todo lo que se desarrolla en las organizaciones campesinas, sus actividades y las vulneraciones de las que son objeto. En este sentido, se consideran la voz de las organizaciones agrarias. Prensa Rural desde su lógica de acción como medio, tiene una relación fuerte con lo indicado por John Downing como medio radical.

http://prensarural.org/spip/

Corporación Memoria y Patrimonio: esta organización desarrolla investigaciones enfocadas en la preservación y conservación de la memoria colectiva en temas de patrimonio. La fortaleza de esta organización han sido sus diversas producciones impresas para la divulgación de las investigaciones. http://memoriaypatrimonio.org/

Corporación Juventud Raizes: es una organización de jóvenes para jóvenes, que busca fortalecer la incidencia política y el análisis crítico de este sector poblacional. Enfocan sus acciones hacia la formación del sujeto político y social. La comunicación que desarrollan está relacionada con la comunicación interpersonal en procesos de capacitación y formación. https://www.facebook.com/corporacionjuventudraizes

Corporación de Objetores y Objetoras por Conciencia-Quinto Mandamiento: proceso organizativo derivado de un acompañamiento externo ofrecido por grupos de mujeres y el movimiento juvenil. Promueve la objeción de conciencia a la prestación del servicio militar obligatorio en Colombia. Apelando al quinto mandamiento "no matarás", esta organización basa su discurso en la no violencia y el antimilitarismo; por ello, en sus acciones de comunicación, se han apoyado en el arte, la música y la protesta social para manifestarse en contra del reclutamiento o las populares batidas $^{6}$. En la actualidad, la corporación se encuentra en tiempo de receso debido a dificultades tributarias. http://www. objetoresquintomandamiento.blogspot.com/

\section{Organizaciones caracterizadas en el municipio de Bucaramanga, área metropolitana}

Comité Cultural de Zapamanga-Cocuza: creada en 1979 como un comité de vecinos producto de una iniciativa parroquial en el auge de la teología de la liberación. Cocuza es una asociación que centra su acción en el barrio Zapamanga del municipio de Floridablanca; no obstante, sus acciones han beneficiado a los habitantes de la comuna 4 de este municipio. Históricamente, Cocuza ha enfocado su trabajo en tres líneas transversales: comunicación e investigación; organización; y participación. En este sentido, para la organización la comunicación se asume como la base del ejercicio político fundamentado en la educación popular, de ahí que su concepto se relaciona con el de estrategia y capacidad para la incidencia.

https://www.facebook.com/comite.zapamanga 
Corporación Buen Ambiente-Corambiente: es una organización que tiene como objeto social el fortalecimiento de la organización campesina, por lo cual se dedican a la promoción de prácticas agropecuarias adecuadas, basadas en cultivos orgánicos. Así mismo, realizan un acompañamiento social y político a las organizaciones campesinas y velan por la promoción de la seguridad alimentaria entre estas familias. (No tienen recursos web).

Corporación para el Desarrollo del Oriente-Compromiso: es una de las organizaciones más representativas del oriente colombiano con sus procesos de defensa de los derechos humanos, el acompañamiento a las víctimas del conflicto armado (desplazados) y la promoción de la participación ciudadana. Son adjudicatarios de la licencia de funcionamiento de la emisora comunitaria La Brújula, en el norte de Bucaramanga. http://www.corporacioncompromiso.org/ http:// labrujularadiocomunitaria.blogspot.com/

Fundación CITU Experiencia Local Laboratorio de Proyectos Urbanos: esta organización se propone hacer aportes de análisis, estudio y proyección para el mejoramiento de las condiciones de vida urbana para la ciudadanía. CITU ha establecido el espacio público y el hábitat urbano como líneas de investigación y acción. En CITU, la comunicación se establece a partir de procesos de diálogo y participación. En la actualidad están diseñando su sitio web, pero algunas de sus acciones en comunicación tienen que ver con la estrategia denominada Telegrama Comunitario: http://

telegramacomunitario.wix.com/jovenes\#!

https://es-la.facebook.com/telegrama.comunitario

https://es-es.facebook.com/CituExperienciaLocal

Corporación para la Construcción Participativa de la Salud Pública-Obusinga: es una organización que busca el mejoramiento de las condiciones de salud de las personas, comprendiendo la salud como atención a todos los factores que contribuyen a que la gente esté saludable. Obusinga se ocupa también de problemas alimentarios, por ello desarrolla investigaciones y acciones sobre la canasta alimentaria básica y en general, sobre las condiciones en que se establece la autonomía alimentaria. Asumen la Investigación-Acción Participativa (IAP) como metodología de trabajo, de ahí que sus acciones de comunicación se soportan en la participación. Como acciones de movilización social en torno al tema de autonomía alimentaria, han desarrollado series radiales y el blog de diálogo alimentario, basado en el periodismo alimentario. http://www.obusinga.com/

Fundación Mujer y Futuro: creada en 1988, esta organización surge para fortalecer los procesos de promoción de los derechos de las mujeres, en especial los derechos sexuales y reproductivos. Así mismo, promueve la reflexión comunitaria y ciudadana en torno a la construcción en la región de nuevas feminidades y masculinidades. Durante su trayectoria, Mujer y Futuro ha procurado el uso de herramientas pedagógicas en comunicación, usualmente impresas y radiales. http:// mujeryfuturo.org/

Corporación para la equidad, la democracia y el buen vivir-Hypatia: enfocada en el tema de los procesos de mujeres, esta corporación amplía su objeto social a la defensa de los derechos humanos de las minorías sexuales y étnicas; la formación de ciudadanía, y la formación de una sociedad más democrática y participativa. http://corpohypatia.org 